\title{
Degradation analysis based on an extended inverse Gaussian process model with skew-normal random effects and measurement errors
}

\author{
Songhua Hao ${ }^{1}$, Jun Yang ${ }^{1 *}$, Christophe Berenguer ${ }^{2}$ \\ ${ }^{1}$ School of Reliability and Systems Engineering, Beihang University, Beijing, China \\ ${ }^{2}$ Univ. Grenoble Alpes, CNRS, Grenoble INP, GIPSA-lab, Grenoble, France
}

\begin{abstract}
As an important degradation model for monotonic degradation processes, the inverse Gaussian (IG) process model has attracted a lot of attention. To characterize random effects among test samples, the traditional IG process model usually assumes a normal distributed degradation rate. However, the degradation rates in some applications may manifest some asymmetric and non-normal behaviors, such as the GaAs laser degradation data. Therefore, we propose an extended inverse Gaussian (EIG) process model by incorporating skew-normal random effects, and derive its analytical lifetime distribution. Furthermore, considering that available studies about IG process models are limited on the aspect of measurement errors, parameter estimation methods for the proposed degradation model are developed for two scenarios, i.e., the maximum likelihood estimations (MLEs) for perfect measurements, and an extended Monte Carlo (MC) integration algorithm for the MLEs for perturbed measurements. Then a simulation study is adopted to show the effectiveness of the proposed MLEs, and two illustrative examples of GaAs laser degradation and fatigue crack growth are provided to illustrate the advantages of the proposed EIG process model, i.e., the improvement in degradation data fitting performance and lifetime evaluation accuracy by incorporating skew-normal random effects and measurement errors.
\end{abstract}

Key words: Extended inverse Gaussian process model; Skew-normal random effects; Measurement errors; the MLE method; Extended MC integration algorithm.

\section{Introduction}

Recently, longevity and high-reliability characteristics have become increasingly important for many systems, which will certainly enhance the difficulty of generating enough failure data in limited-duration reliability tests, and

* Corresponding author. Tel.: +86 1082316003 ; fax: +86 1082316003 . 
therefore pose challenges to reliability estimation by traditional lifetime-based methods. In these circumstances, degradation analysis has been proposed as an alternative approach to reliability assessment, with the advantages of requiring smaller sample sizes and shorter test durations [1]. A lot of studies have contributed to the development and extension of degradation models, which can be referred to in the recent review papers in [2] and [3].

Because of the necessity to capture stochastic dynamics in degradation phenomena, modelling approaches based stochastic processes have developed as a preferred and promising option for degradation analysis. And, in particular, the Wiener process, the Gamma process and the inverse Gaussian (IG) process are three popular classes of stochastic degradation processes for continuous degradation phenomena modelling. Thanks to its mathematical tractability, the Wiener process has been under extensive studies and has been applied to many non-monotonical degradation processes $[4,5]$. For monotonic and gradual degradation processes like crack growth, the Gamma process is an alternative and appropriate model [6,7].

However, the Gamma process does not work well for all monotonic degradation processes. For the GaAs laser degradation data in [1], Wang and Xu showed the poor fitting effects of Wiener and Gamma processes, and proposed an inverse Gaussian (IG) process model with better fitting results [8]. Since then, the IG process has become an important supplement to the stochastic degradation processes toolbox. Ye and Chen illustrated the physical interpretation of the IG process, as well as its inverse relation with the Wiener process [9]. Further researches on the IG process model include accelerated degradation planning [10], condition-based replacement and inspection policy optimization[11], remaining useful life estimation [12], bivariate degradation analysis [13,14], reliability analysis for multicomponent systems under dynamic environments [15] and reliability modelling with random initial degradation [16]. Particularly, Guo et al. considered that the degradation increments depend on the current degradation state, and proposed an improved IG process[17]. To account for model uncertainty during monotonic degradation modeling, Liu et al. developed a Bayesian averaging method for the inverse Gaussian process and the Gamma process [18]. Besides, many studies have been devoted to degradation modelling approaches based on the IG process from a Bayesian perspective [19-23].

Generally, the heterogeneity among test samples leads to unit-to-unit variability in degradation paths, and this calls for the development of degradation models with random effects [24]. A traditional way to incorporate random effects in the model is to assume some normal distributed model parameters, which can be seen in [25-27], respectively for the Wiener process, the Gamma process and the IG process. However, this assumption may not be appropriate in all applications. In fact, the traditional normal distribution is symmetric, but the degradation rates in 
some applications may manifest asymmetry and non-normal behaviors [28,29]. The skew-normal distribution, including the normal distribution as a special case, has been proven appropriate for many applications with asymmetric behaviors [30-32], and is recently recommended to model the random effects in degradation processes [33]. Peng and Tseng analyzed the previously mentioned GaAs laser degradation data, and indicated that the skewnormal distribution fits the random effects better than the normal distribution [34]. Skew-normal random effects have been also incorporated to develop the generalized Wiener process model[35], the adaptive skew-Wiener process model [36] and the nonlinear skew-Wiener degradation model [29].

Besides random effects, measurement errors constitute another source of variability contributing to the uncertainty of degradation processes, and pose a great challenge to model parameter estimation. Confronted with this issue, a common assumption is to consider the measurement error as a zero-mean normally distributed variable. For example, Pan et al. proposed an expectation maximization algorithm for time-transformed Wiener processes with temporal variability, unit-to-unit heterogeneity and measurement errors [37]. To calculate the likelihood function for the Gamma process model under imperfect inspection, Kallen and Noortwijk proposed a general Monte Carlo (MC) integration algorithm with convergence rate $\mathrm{O}\left(N^{-1 / 2}\right)$, where $N$ is the number of repetition [38]. Lu, Pandey and Xie provided a clearer explanation and formulation of the MC approach [39], which is also applied to a novel Gamma process with measurement errors that statistically depend on the temporal degradation performance [40].

To the best of our knowledge, the existing researches about IG processes only consider normal random effects. However, for the GaAs laser degradation data that motivated to propose the IG process model, it has been proven that the skew-normal distribution fits the random effects better [34]. Furthermore, studies about the IG process model with measurement errors remain limited, except for two corrosion depth growth models for energy pipelines in [41,42], which did not cover the issue of random effects. Therefore, in this paper we extend the existing approaches of the IG stochastic degradation process by considering at the same time skew-normal random effects (which includes normal random effects as a special case) and measurements errors. Our contribution thus consists in the proposition of a degradation model based on an IG process with asymmetric random effects and in the development of the associated parameter estimation methods for both perfect measurements and perturbed measurements.

The remaining of the paper is organized as follows. In Section 2, we use the GaAs laser degradation data as a motivating example of this work, and further propose an EIG process model with skew-normal random effects. Section 3 evaluates the analytical failure time distribution of the proposed EIG process model. Degradation model parameter estimation methods for both perfect measurements and perturbed measurements are developed in Section 
4. Section 5 illustrates the advantages of incorporating skew-normal random effects and measurement errors, based on a simulation study and two applications of GaAs laser and fatigue crack growth. Section 6 concludes the paper with some remarks and perspective on future work.

\section{An EIG process model with skew-normal random effects}

\subsection{Motivating example}

In this section, we revisit the mentioned GaAs laser degradation data, and illustrate the necessity to consider skew-normal random effects for the IG process model.

The laser device degradation data was provided by Meeker and Escobar in [1], and has been studied by many researchers. In general, the output light intensity will degrade over time, but in order to obtain constant laser output, a feedback mechanism is adapted to increase the operating current, and thus transferring the output degradation to input degradation. The time-varying operating current degradation paths of $15 \mathrm{GaAs}$ laser device samples are provided in [1] and plotted in Figure 1. The current of the samples was measured every 250 hours, and the test was terminated at 4000 hours. The product is declared as failed when the input current exceeds 10A.

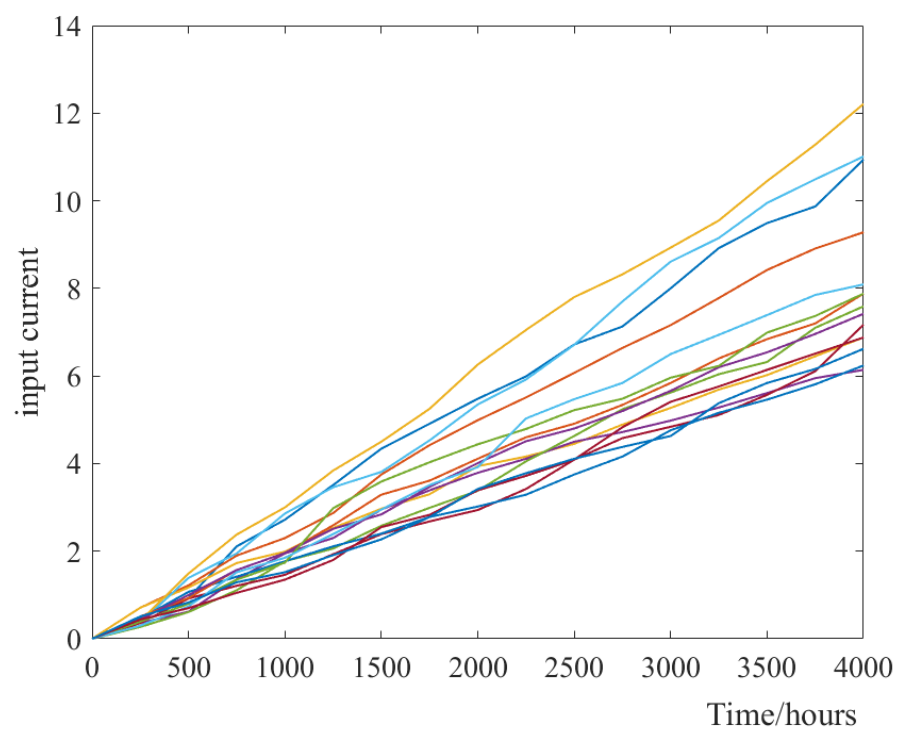

Figure 1 Degradation paths of $15 \mathrm{GaAs}$ laser samples

To describe this set of GaAs laser degradation data of, various models have been developed. For example, in [43], Peng and Tseng proposed a Wiener process model with normal random effects. And in [27], Peng further presented an IG process model with normal random effects, and illustrated its advantage over the Wiener process model by Akaike information criterion (AIC). Recently in [34], it is illustrated that a skew-normal distribution is more appropriate to fit the slopes of the laser degradation data, and the proposed skew-Wiener process model has 
better performance than the conventional Wiener process model with normal random effects. Therefore, it is motivated to also extend the IG process model by considering skew-normal random effects.

Ye and Chen have demonstrated that there is an inverse relation between the IG and Wiener processes [9], i.e., a Wiener process $W(x)=\beta^{-1} x+\eta^{-1 / 2} t B(x)$ for the degradation will induce an IG process $Y(t) \sim I G\left(\beta \Lambda(t), \eta \Lambda^{2}(t)\right)$ on the first passage times of the threshold $\Lambda(t)$, and this motivates to incorporate random effects in the IG process by letting $\beta^{-1}$ follow a normal distribution, which is the case in most exiting works about the IG process. Therefore, instead of investigating the distribution characteristic of the degradation slopes, we reanalyze the inverse slopes in the following.

For the laser degradation data of 15 test samples, a regression analysis is conducted to obtain 15 positive slopes, then we can also obtain 15 inverse slopes. Similar to the candidates of distribution types for random effects in [34], here we also fit the inverse slopes with the Normal, Gamma, Log-Normal, Weibull and Skew-normal distributions. Maximum likelihood estimations (MLEs) for the parameters of each distribution are calculated, with the loglikelihood listed in Table 1. Furthermore, we select the best distribution type for random effects based on the popular AIC index, which is denoted as follows:

$$
A I C=-2 \ln (\hat{L})+2 k
$$

where $\hat{L}$ is the maximum value of the log-likelihood function for the candidate distribution, and $k$ is the number of distribution parameters. Generally, the distribution type with the smallest AIC will be chosen as the best model.

Table 1 lists the AIC for each distribution type for random effects. These results show that the skew-normal distribution, with the smallest AIC, leads to the best fitting performance on the inverse slopes of the laser data. Therefore, based on the skew-normal random effects, we propose an EIG process model in the following subsection.

Table 1 Maximum log_likelihoods and AICs for the inverse slopes of laser degradation data

\begin{tabular}{|c|c|c|}
\hline Distribution type for random effects & $\hat{L}$ & AIC \\
\hline Normal & -89.8168 & 183.6336 \\
\hline Gamma & -90.3049 & 184.6098 \\
\hline Lognormal & -90.6636 & 185.3272 \\
\hline Weibull & -89.1474 & 182.2948 \\
\hline Skew-normal & $\mathbf{- 8 7 . 4 8 9 2}$ & $\mathbf{1 7 8 . 9 7 8 4}$ \\
\hline
\end{tabular}




\subsection{Model assumption and formulation}

Defined as a stochastic degradation process, an IG process $\left\{Y_{0}(t), t \geq 0\right\}$ with fixed effects satisfies the following properties:

(1) $Y_{0}(0)=0$ with probability 1 ;

(2) Increments of $Y_{0}(t)$ are independent, that is, $Y_{0}\left(t_{2}\right)-Y_{0}\left(t_{1}\right)$ is independent with $Y_{0}\left(t_{4}\right)-Y_{0}\left(t_{3}\right)$ for $\forall t_{4}>t_{3} \geq t_{2}>t_{1} \geq 0$

(3) $Y_{0}(t)-Y_{0}(s)$ follows an IG distribution $I G\left(\beta \Delta \Lambda(t), \eta \Delta \Lambda^{2}(t)\right)$ for $\forall t>s \geq 0$, where $\beta$ and $\eta$ are respectively the slope and shape parameters, $\Lambda(t)$ is the monotonously increasing drift function.

Therefore, $Y_{0}(t)$ follows an IG distribution $I G\left(\beta \Lambda(t), \eta \Lambda^{2}(t)\right)$, with the following probability density function (PDF) and cumulative distribution function (CDF):

$$
\begin{gathered}
f_{Y_{0}}(y)=\sqrt{\frac{\eta \Lambda^{2}(t)}{2 \pi y^{3}}} \exp \left\{-\frac{\eta(y-\beta \Lambda(t))^{2}}{2 \beta^{2} y}\right\} \\
F_{Y_{0}}(y)=\Phi\left[\sqrt{\frac{\eta \Lambda^{2}(t)}{y}}\left(\frac{y}{\beta \Lambda(t)}-1\right)\right]+\exp \left\{\frac{2 \eta \Lambda(t)}{\beta}\right\} \Phi\left[-\sqrt{\frac{\eta \Lambda^{2}(t)}{y}}\left(\frac{y}{\beta \Lambda(t)}+1\right)\right]
\end{gathered}
$$

where $\Phi()$ is the CDF of standard normal distribution.

To incorporate random effects in the IG process model, the traditional way is to let $\delta=\beta^{-1}$ follow a normal distribution. Here by relaxing the traditional normal assumption to a skew-normal one, we propose an EIG process model, where the PDF of $\delta=\beta^{-1} \sim S N\left(\mu, \sigma^{2}, \alpha\right)$ is given by

$$
f_{\delta}(x)=\frac{2}{\sigma} \phi\left(\frac{x-\mu}{\sigma}\right) \Phi\left(\alpha \frac{x-\mu}{\sigma}\right)
$$

where $\mu, \sigma, \alpha$ are respectively the location, scale and shape parameters, $\phi()$ is the PDF of standard normal distribution.

Note that if $\alpha=0, S N\left(\mu, \sigma^{2}, \alpha\right)$ will reduce to a normal distribution, which indicates that the traditional IG process model with normal random effects is a special case of the proposed EIG process model. 


\section{Reliability assessment and lifetime evaluation}

After developing the EIG process model, a significant task in reliability engineering is to assess the reliability and lifetime. A degrading system is declared as failed when its degradation path first exceeds a predetermined threshold level $D$, and due to the monotonicity property of the IG process, its lifetime CDF can be expressed as

$$
F_{T}(t)=P\{Y(t)<D\}
$$

For the traditional IG process model with fixed effects, the CDF of lifetime $T_{0}$ can be derived as [8]:

$$
F_{T_{0}}(t)=P\left\{Y_{0}(t)<D\right\}=F_{Y_{0}}(D)=\Phi\left[\sqrt{\frac{\eta \Lambda^{2}(t)}{D}}\left(\frac{D}{\beta \Lambda(t)}-1\right)\right]+\exp \left\{\frac{2 \eta \Lambda(t)}{\beta}\right\} \Phi\left[-\sqrt{\frac{\eta \Lambda^{2}(t)}{D}}\left(\frac{D}{\beta \Lambda(t)}+1\right)\right]
$$

where $T_{0}$ is the lifetime for the IG process model with fixed effects.

For the IG process model with normal random effects, the CDF of lifetime $T_{1}$ can be derived by integrating $F_{T_{0}}(t)$ with respect to $\beta^{-1} \sim N\left(\mu, \sigma^{2}\right)[12]:$

$$
F_{T_{1}}(t)=\int_{-\infty}^{+\infty} F_{T_{0}}(t \mid u) \phi\left(\frac{u-\mu}{\sigma}\right) d u=\Phi\left(\sqrt{\frac{\eta}{D}} \frac{\sigma \Lambda(t)-\mu \sigma D}{\sqrt{\sigma^{2}+\eta D}}\right)-e^{2 \mu \eta \Lambda(t)+\frac{2 \eta^{2} \Lambda^{2}(t)}{\sigma^{2}}} \Phi\left(-\sqrt{\frac{\eta}{D}} \frac{\left(\sigma^{2}+2 \eta D\right) \Lambda(t)+\mu \sigma^{2} D}{\sqrt{\sigma^{4}+\eta D \sigma^{2}}}\right)
$$

where $T_{1}$ is the lifetime for the IG process model with normal random effects, and $F_{T_{0}}(t \mid u)$ is the CDF of $T_{0}$ with fixed $\beta=u^{-1}$.

For the IG process model with skew-normal random effects, the derivation of the CDF of the lifetime $T_{2}$ can be derived by integrating $F_{T_{0}}(t)$ with respect to $\delta=\beta^{-1} \sim S N\left(\mu, \sigma^{2}, \alpha\right)$. The derivation process is a little tricky, so in order to clarify the process, we first develop the following Lemmas 1-3 and Theorem 1.

Lemma 1. if $a_{1}, b_{1}, a_{2}, b_{2} \in R$, then the following result holds,

$$
\int_{-\infty}^{+\infty} \Phi\left(a_{1}+b_{1} x\right) \Phi\left(a_{2}+b_{2} x\right) \phi(x) d x=\Phi_{2}\left(\frac{a_{1}}{\sqrt{1+b_{1}^{2}}}, \frac{a_{2}}{\sqrt{1+b_{2}^{2}}}, \frac{b_{1} b_{2}}{\sqrt{\left(1+b_{1}^{2}\right)\left(1+b_{2}^{2}\right)}}\right)
$$

where $\Phi_{2}(\cdot)$ denotes the CDF of the standard bivariate normal distribution:

$$
\Phi_{2}\left(k_{1}, k_{2}, \rho\right)=\frac{1}{2 \pi \sqrt{1-\rho^{2}}} \int_{-\infty}^{k_{1}} \int_{-\infty}^{k_{2}} \exp \left\{-\frac{x^{2}-2 \rho x y+y^{2}}{2\left(1-\rho^{2}\right)}\right\} d x d y
$$

where $k_{1}, k_{2} \in R$ and $-1<\rho<1$. 
Although Owen has given the same result as early as 1980 [44], the proof was omitted. In order to fulfil this gap, here we present the proof of Lemma 1 in Appendix A, relying on the Dominated Convergence Theorem.

Lemma 2. If $V \sim S N\left(\mu, \sigma^{2}, \alpha\right)$ and $A, B, C \in R$, then the following result holds:

$$
E_{V}[\Phi(A+B V)]=2 \Phi_{2}\left(\frac{A+B \mu}{\sqrt{1+B^{2} \sigma^{2}}}, 0, \frac{B \alpha \sigma}{\sqrt{\left(1+B^{2} \sigma^{2}\right)\left(1+\alpha^{2}\right)}}\right)
$$

Proof: According to the result of Lemma 1, along with some changes of variables, the proof of the above result is rather straightforward, and is thus omitted here.

Lemma 3. If $V \sim S N\left(\mu, \sigma^{2}, \alpha\right)$ and $A, B, C \in R$, then the following result holds:

$$
E_{V}\left[e^{C V} \Phi(A+B V)\right]=2 e^{C \mu+\frac{C^{2} \sigma^{2}}{2}} \Phi_{2}\left(\frac{A+B \mu+B C \sigma^{2}}{\sqrt{1+B^{2} \sigma^{2}}}, \frac{B \sigma \alpha}{\sqrt{\left(1+\alpha^{2}\right)}}, \frac{B \alpha \sigma}{\sqrt{\left(1+B^{2} \sigma^{2}\right)\left(1+\alpha^{2}\right)}}\right)
$$

Proof: Based on some algebraic manipulations, we can obtain:

$$
\begin{aligned}
& E_{V}\left[e^{C V} \Phi(A+B V)\right] \\
= & \int_{-\infty}^{+\infty} e^{C v} \Phi(A+B v) \frac{2}{\sqrt{2 \pi} \sigma^{2}} \Phi\left(\alpha \frac{v-\mu}{\sigma}\right) e^{-(v-\mu)^{2} / 2 \sigma^{2}} d v \\
= & \frac{2}{\sqrt{2 \pi} \sigma^{2}} \int_{-\infty}^{+\infty} \Phi(A+B v) \Phi\left(\alpha \frac{v-\mu}{\sigma}\right) \exp \left\{-\frac{\left[v-\left(\mu-C \sigma^{2}\right)^{2}\right]}{2 \sigma^{2}}+C \mu+\frac{C^{2} \sigma^{2}}{2}\right\} d v \\
= & \frac{2}{\sigma} e^{C \mu+\frac{C^{2} \sigma^{2}}{2}} \int_{-\infty}^{+\infty} \Phi(A+B v) \Phi\left(\alpha \frac{v-\mu}{\sigma}\right) \phi\left(\frac{v-\left(\mu+C^{2} \sigma^{2}\right)}{\sigma}\right) d v \\
= & \frac{2}{\sigma} e^{C \mu+\frac{C^{2} \sigma^{2}}{2}} \int_{-\infty}^{+\infty} \Phi\left(A+B \mu+B C^{2} \sigma^{2}+B \sigma v^{\prime}\right) \Phi\left(\alpha C^{2} \sigma+\alpha v^{\prime}\right) \phi\left(v^{\prime}\right) \cdot \sigma d v^{\prime}
\end{aligned}
$$

where $v^{\prime}=\frac{v-\left(\mu+C^{2} \sigma^{2}\right)}{\sigma}$.

Therefore, with some changes of variables, Equation (12) can be established based on the result of Lemma 1.

Theorem 1. For the IG process model with skew-normal random effects, the CDF of lifetime $T_{2}$ is:

$$
\begin{aligned}
F_{T_{2}}(t) & =2 \Phi_{2}\left(\frac{\sqrt{\eta}(\mu D-\Lambda(t))}{\sqrt{D+\eta D^{2} \sigma^{2}}}, 0, \frac{\sqrt{\eta D} \alpha \sigma}{\sqrt{\left(1+\eta D \sigma^{2}\right)\left(1+\alpha^{2}\right)}}\right)+2 \exp \left\{2 \eta \mu \Lambda(t)+2 \eta^{2} \sigma^{2} \Lambda^{2}(t)\right\} \\
& \times \Phi_{2}\left(\frac{-\sqrt{\eta}\left(\Lambda(t)+\mu D+2 \eta D \sigma^{2} \Lambda(t)\right)}{\sqrt{D+\eta D^{2} \sigma^{2}}}, \frac{-\sqrt{\eta D} \sigma \alpha}{\sqrt{1+\alpha^{2}}}, \frac{-\sqrt{\eta D} \alpha \sigma}{\sqrt{\left(1+\eta D \sigma^{2}\right)\left(1+\alpha^{2}\right)}}\right)
\end{aligned}
$$


Proof: According to the formula of total probability, the CDF of $T_{2}$ can be derived by integrating $F_{T_{0}}(t)$ with respect to $\delta=\beta^{-1} \sim S N\left(\mu, \sigma^{2}, \alpha\right)$ :

$$
F_{T_{2}}(t)=\int_{-\infty}^{+\infty} F_{T_{0}}(t \mid \delta) f_{\delta}(x) d x=E_{\delta}\left[\Phi\left(-\sqrt{\frac{\eta \Lambda^{2}(t)}{y}}+\sqrt{\eta y} \delta\right)\right]+E_{\delta}\left[e^{2 \eta \Lambda(t) \delta} \Phi\left(-\sqrt{\frac{\eta \Lambda^{2}(t)}{y}}-\sqrt{\eta y} \delta\right)\right]
$$

where $E_{\delta}()$ is the expectation of functions of $\delta$.

Therefore, respectively calculating the two expectations based on Lemma 2 and Lemma 3, we can obtain Equation (13).

\section{Degradation model parameter estimation}

In this section, we focus on estimating the EIG model parameters based on the degradation data of a practical system. Two MLEs are developed for two scenarios of measured degradation performance, including perfect measurements and perturbed measurements.

\subsection{MLEs for perfect measurements}

We first consider the scenario of perfect measurements. Suppose a total of $N$ samples are tested under identical condition, and the degradation of them is perfectly measured at ordered times $t_{1}, t_{2}, \cdots, t_{M}$, where $M$ denotes the available number of measurements. For $n=1,2, \cdots, N$ and $m=1,2, \cdots, M$, let $y_{n, m}=y\left(t_{n, m}\right)$ denote the degradation performance of sample $n$ at time $t_{m}$. Furthermore, denote the transformed time interval and degradation increment by $T_{m}=\Lambda\left(t_{m}\right)-\Lambda\left(t_{m-1}\right)$ and $\Delta y_{n, m}=y_{n, m}-y_{n, m-1}$, where $t_{0}=0, y_{n, 0}=0$ holds for all $n$.

Due to the independence between degradation increments, the complete log-likelihood function is derived as:

$$
L(\mathbf{x})=\sum_{n=1}^{N} \sum_{m=1}^{M} \ln f_{\Delta Y}\left(\Delta y_{n, m}\right)
$$

where $f_{\Delta Y}(\cdot)$ is the complete PDF of degradation increment considering skew-normal random effects, which can be derived by integrating the PDF in Equation (2) with respect to $\delta=\beta^{-1} \sim S N\left(\mu, \sigma^{2}, \alpha\right)$, and to clarify the derivation process, a theorem is first provided:

Theorem 2. If $Z \sim S N\left(\mu, \sigma^{2}, \alpha\right)$ and $A, B \in R$, then the following result holds: 


$$
E_{Z}\left[\exp \left(A Z+B Z^{2}\right)\right]=\frac{2}{\sqrt{1-2 B \sigma^{2}}} \exp \left(\frac{2 B \mu^{2}+A^{2} \sigma^{2}+2 A \mu}{2\left(1-2 B \sigma^{2}\right)}\right) \times \Phi\left(\frac{\alpha \sigma(A+2 B \mu)}{\left(1-2 B \sigma^{2}\right)^{2}+\alpha^{2}\left(1-2 B \sigma^{2}\right)}\right)
$$

The proof of Theorem 2 is given in Appendix B.

Based on the given Theorem 2 and some changes of variables, $f_{\Delta Y}(\cdot)$ can be expressed by:

$$
\begin{aligned}
f_{\Delta Y}(y) & =\int_{-\infty}^{+\infty} f_{\Delta Y_{0}}\left(y \mid \delta=\beta^{-1}=x\right) f_{\delta}(x) d x \\
& =E_{\delta}\left[\sqrt{\frac{\eta \Delta \Lambda^{2}(t)}{2 \pi y^{3}}} \exp \left\{-\frac{\eta}{2 y}\left[y^{2} \delta^{2}-2 \Delta \Lambda(t) y \delta+\Delta \Lambda^{2}(t)\right]\right\}\right] \\
& =\sqrt{\frac{\eta \Delta \Lambda^{2}(t)}{2 \pi y^{3}}} \exp \left\{-\frac{\eta \Delta \Lambda^{2}(t)}{2 y}\right\} E_{\delta}\left[\exp \left(\eta \Delta \Lambda(t) \delta-\frac{\eta y}{2} \delta^{2}\right)\right] \\
& =\sqrt{\frac{2 \eta \Delta \Lambda^{2}(t)}{\pi y^{3}\left(1+\eta y \sigma^{2}\right)}} \exp \left(\frac{-\eta y^{2} \mu^{2}+2 \eta \mu y \Delta \Lambda(t)-\eta \Delta \Lambda^{2}(t)}{2 y\left(1+\eta y \sigma^{2}\right)}\right) \times \Phi\left(\frac{\alpha \sigma(\eta \Delta \Lambda(t)-\eta y \mu)}{\left(1+\eta y \sigma^{2}\right)^{2}+\alpha^{2}\left(1+\eta y \sigma^{2}\right)}\right)
\end{aligned}
$$

Therefore, the complete log-likelihood function will be:

$$
\begin{aligned}
L(\mathbf{x})= & \sum_{n=1}^{N} \sum_{m=1}^{M} \ln f_{\Delta Y}\left(\Delta y_{n, m}\right) \\
= & \frac{M N}{2}(\ln 2+\ln \eta-\ln \pi)+N \sum_{m=1}^{M} \ln T_{m}-\frac{3}{2} \sum_{n=1}^{N} \sum_{m=1}^{M} \ln \Delta y_{n, m} \\
& -\frac{1}{2} \sum_{n=1}^{N} \sum_{m=1}^{M} \ln \left(1+\eta \sigma^{2} \Delta y_{n, m}\right)+\sum_{n=1}^{N} \sum_{m=1}^{M}\left[\frac{-\eta \mu^{2} \Delta y_{n, m}^{2}+2 \eta \mu T_{m} \Delta y_{n, m}-\eta T_{m}^{2}}{2 \Delta y_{n, m}\left(1+\eta \Delta y_{n, m} \sigma^{2}\right)}\right] \\
& +\sum_{n=1}^{N} \sum_{m=1}^{M} \ln \left[\Phi\left(\frac{\alpha \sigma\left(\eta T_{m}-\eta \mu \Delta y_{n, m}\right)}{\left(1+\eta \sigma^{2} \Delta y_{n, m}\right)^{2}+\alpha^{2}\left(1+\eta \sigma^{2} \Delta y_{n, m}\right)}\right)\right]
\end{aligned}
$$

Considering that it is rather difficult to directly maximize the above log-likelihood function, evolutionary methods like genetic algorithm (GA) provide an alternative solution. The GA is an intelligent search algorithm, and has been applied to the parameter estimation of many degradation models, e.g., $[24,45,46]$. Therefore, here we also obtain the MLEs of parameters $\boldsymbol{\Theta}=\left\{\boldsymbol{\theta}_{\Lambda}, \eta, \mu, \sigma, \alpha\right\}$ via GA, where $\boldsymbol{\theta}_{\Lambda}$ is the unknown parameters in the drift function $\Lambda(t)$.

\subsection{MLEs for perturbed measurements}

Due to the limitations of measurement instruments or procedures, measurement errors often add uncertainty to the observed degradation process. Therefore, here we present a parameter estimation method for perturbed measurements.

As classically assumed, a normally distributed variable with zero mean is incorporated into the model to describe 
the measurement error item:

$$
Z\left(t_{k}\right)=Y\left(t_{k}\right)+\varepsilon\left(t_{k}\right)
$$

where $Z\left(t_{k}\right)$ is the measured degradation performance at time $t_{k}, \quad Y\left(t_{k}\right)$ is the actual degradation performance at time $t_{k}$, and $\varepsilon\left(t_{k}\right) \sim N\left(0, \sigma_{\varepsilon}^{2}\right)$ is the measurement error item at time $t_{k}$.

Keeping the same notations as in the previous subsection, except that $y_{n, m}$ and $\Delta y_{n, m}$ are denoted for the actual degradation performance, and let $z_{n, m}=z\left(t_{n, m}\right)$ denote the measured degradation performance of sample $n$ at time $t_{m}, \Delta z_{n, m}=z_{n, m}-z_{n, m-1}$ denote the measured degradation increment, where $z_{n, 0}=0$ holds for all $n$.

Considering the MC integration method in [38] can only estimate the parameters not concerning measurement errors, in other words, this method requires the parameter $\sigma_{\varepsilon}$ to be known and aims to estimate the other parameters. Therefore, in order to estimate all parameters together, an extended MC integration algorithm is presented as follows:

(1) Based on the parameter initial guess method in subsection 3.3 of [47], determine an initial guess $\hat{\sigma}_{\varepsilon}$ and a proper searching range $\left[\hat{\sigma}_{\varepsilon}-\Delta, \hat{\sigma}_{\varepsilon}+\Delta\right]$ for parameter $\sigma_{\varepsilon}$;

(2) Take a positive integer $I$ and an equally spaced sequence $\left(\sigma_{\varepsilon}^{1}, \sigma_{\varepsilon}^{2}, \cdots, \sigma_{\varepsilon}^{I}\right)$, where $\sigma_{\varepsilon}^{i}=\sigma_{\varepsilon}-\Delta+\frac{2 \Delta(i-1)}{I-1}$ holds for $i=1,2, \cdots, I$;

(3) For each $\sigma_{\varepsilon}^{i}, i=1,2, \cdots, I$, generate $J$ independent samples of normally distributed measurement error with mean zero and standard deviation $\sigma_{\varepsilon}^{i}$, and denoted them by $\boldsymbol{E}_{1}^{i}, \boldsymbol{E}_{2}^{i}, \cdots, \boldsymbol{E}_{J}^{i}$, where $\boldsymbol{E}_{j}^{i}=\left\{\varepsilon_{1, j}^{i}, \varepsilon_{2, j}^{i}, \cdots, \varepsilon_{M, j}^{i}\right\}$ holds for $j=1,2, \cdots, J$, and $M$ denotes the available number of measurements;

(4) Compute the measurement error increments as $\Delta \boldsymbol{E}_{1}^{i}, \Delta \boldsymbol{E}_{2}^{i}, \cdots, \Delta \boldsymbol{E}_{J}^{i}$, where $\Delta \boldsymbol{E}_{j}^{i}=\left\{\varepsilon_{1, j}^{i}, \varepsilon_{2, j}^{i}-\varepsilon_{1, j}^{i}, \cdots, \varepsilon_{M, j}^{i}-\varepsilon_{M-1, j}^{i}\right\} ;$

(5) Calculate the simulated actual degradation increments as $\Delta y_{n, m, j}^{i}=\Delta y_{n, m}-\Delta \varepsilon_{m, j}^{i}$, where $\Delta \varepsilon_{m, j}^{i}$ is the $m$ th element of vector $\Delta \boldsymbol{E}_{j}^{i}$;

(6) For Equation (18), replace the degradation increment $\Delta y_{n, m}$ by the new simulated $\Delta y_{n, m, j}^{i}$, and then apply the GA to obtain the MLEs of parameters $\hat{\boldsymbol{\Theta}}_{j}^{i}$ and the corresponding maximum log-likelihood function value $L_{j}^{i}$; 
(7) Therefore, on condition that $\hat{\sigma}_{\varepsilon}=\sigma_{\varepsilon}^{i}$, the MLEs of unknown parameters and the corresponding maximum log-likelihood function value are respectively expressed as:

$$
\begin{gathered}
\hat{\boldsymbol{\Theta}}^{i}=\frac{1}{J} \sum_{j=1}^{J} \hat{\boldsymbol{\Theta}}_{j}^{i} \\
L^{i}=\frac{1}{J} \sum_{j=1}^{J} L_{j}^{i}
\end{gathered}
$$

(8) Find the largest item among all $L^{i}, i=1,2, \cdots, I$, i.e., $L^{k}=\max \left\{L^{1}, L^{2}, \cdots, L^{I}\right\}$, then the parameter estimation result will be $\left\{\hat{\boldsymbol{\Theta}}, \hat{\sigma}_{\varepsilon}\right\}=\left\{\hat{\boldsymbol{\Theta}}^{k}, \sigma_{\varepsilon}^{k}\right\}$.

\section{Numerical experiments: degradation and reliability model verification}

In this section, a simulation study is presented to show the effectiveness of the proposed parameter estimation methods, and discuss the effect of model mis-specification on lifetime estimation accuracy. Besides, two real applications are provided to demonstrate the advantages of the proposed EIG process model. The mentioned GaAs laser example is revisited to illustrate the necessity of incorporating skew-normal random effects, and the example of fatigue crack growth is conducted to show the advantage of considering measurement errors.

\subsection{Simulation study}

At first, Monte Carlo simulations are conducted to study the performance of the proposed MLEs for the EIG process model. Both cases of perfect measurements and perturbed measurements are under consideration. Without loss of generality, we focus on the EIG process model with power drift function, i.e., $\Lambda(t)=t^{b}$. In the simulations, the sample size is set to be $n=30,60,90$, and the degradation parameters are $\mu=2, \sigma=0.2, \alpha=5, \eta=2, b=1.2$. For each sample, we generate its degradation path with inspection interval $\Delta t=1$, and the number of inspections is 50. Based on the simulated sample degradation data and the proposed parameter estimation methods, we can obtain the MLEs for the proposed EIG process model. For both cases, the simulations are repeated 1000 times to obtain the bias and standard deviations of the MLEs.

The parameter estimation results are shown in Tables 2-3. It can be indicated that through the developed MLE methods, the proposed EIG process model parameters can be accurately estimated. Besides, for both cases of perfect measurements and perturbed measurements, the biases and standard deviations of the MLEs will decrease as the sample size increases. And although the contamination of measurement errors will lead to larger biases and standard 
deviations, the MLEs still show rather good performance for all model parameters.

Table 2 Bias (standard deviation) of the MLEs for the EIG process model with skew-normal random effects

\begin{tabular}{|c|c|c|c|c|c|}
\hline$n$ & $\mu=2$ & $\sigma=0.2$ & $\alpha=5$ & $\eta=2$ & $b=1.2$ \\
\hline 30 & $\begin{array}{c}0.0747 \\
(0.1441)\end{array}$ & $\begin{array}{c}-0.0201 \\
(0.0343)\end{array}$ & $\begin{array}{c}0.0261 \\
(0.2459)\end{array}$ & $\begin{array}{c}0.0143 \\
(0.2655)\end{array}$ & $\begin{array}{c}0.0035 \\
(0.0174)\end{array}$ \\
\hline \multirow{2}{*}{60} & $\begin{array}{c}0.0713 \\
(0.1435)\end{array}$ & $\begin{array}{c}-0.0149 \\
(0.0271)\end{array}$ & $\begin{array}{c}0.0205 \\
(0.2430)\end{array}$ & $\begin{array}{c}0.0119 \\
(0.2620)\end{array}$ & $\begin{array}{c}0.0032 \\
(0.0161)\end{array}$ \\
\hline \multirow{2}{*}{90} & $\begin{array}{c}0.0460 \\
(0.1397)\end{array}$ & $\begin{array}{c}-0.0128 \\
(0.0264)\end{array}$ & $\begin{array}{c}0.0235 \\
(0.2553)\end{array}$ & $\begin{array}{c}0.0078 \\
(0.2517)\end{array}$ & $\begin{array}{c}0.0015 \\
(0.0162)\end{array}$ \\
\hline
\end{tabular}

Table 3 Bias (standard deviation) of the MLEs for the EIG process model with both skew-normal random effects and measurement errors

\begin{tabular}{|c|c|c|c|c|c|c|}
\hline \multirow{2}{*}{$n$} & $\mu=2$ & $\sigma=0.2$ & $\alpha=5$ & $\eta=2$ & $b=1.2$ & $\sigma_{\varepsilon}=1$ \\
\hline \multirow{2}{*}{30} & $\begin{array}{c}-0.1717 \\
(0.1770)\end{array}$ & $\begin{array}{c}0.0235 \\
(0.0305)\end{array}$ & $\begin{array}{c}0.0978 \\
(0.2047)\end{array}$ & $\begin{array}{c}-0.0597 \\
(0.1628)\end{array}$ & $\begin{array}{c}0.1021 \\
(0.1518)\end{array}$ & $\begin{array}{c}0.0350 \\
(0.1664)\end{array}$ \\
\hline \multirow{2}{*}{60} & -0.1681 & 0.0195 & 0.0467 & -0.0433 & 0.0582 & 0.0594 \\
& $(0.1706)$ & $(0.0337)$ & $(0.2259)$ & $(0.1941)$ & $(0.1692)$ & $(0.2010)$ \\
\hline \multirow{2}{*}{90} & -0.1453 & 0.0141 & 0.0449 & -0.0285 & -0.0196 & -0.0184 \\
& $(0.2065)$ & $(0.0390)$ & $(0.2401)$ & $(0.1961)$ & $(0.1610)$ & $(0.2667)$ \\
\hline
\end{tabular}

Furthermore, we investigate the effect of model mis-specification on the performance of the parameter estimation and reliability assessment. To this aim, we generate simulated degradation data using the proposed EIG process model, for both cases of perfect measurements and perturbed measurements, with the same model parameters as in the previous example and with a sample size 90 . Then the simulated samples are used for parameter estimations for the proposed EIG model and for different available IG process models. These "candidate" degradation models are:

- IG process model with fixed effect (IG_FE):

$$
Y(t) \sim I G\left(\beta \Lambda(t), \eta \Lambda^{2}(t)\right), \beta^{-1}=\mu
$$

- IG process model with normal random effects (IG_NRE):

$$
Y(t) \sim I G\left(\beta \Lambda(t), \eta \Lambda^{2}(t)\right), \beta^{-1} \sim N\left(\mu, \sigma^{2}\right)
$$

- IG process model with skew-normal random effects (IG_SNRE):

$$
Y(t) \sim I G\left(\beta \Lambda(t), \eta \Lambda^{2}(t)\right), \beta^{-1} \sim S N\left(\mu, \sigma^{2}, \alpha\right)
$$

- IG process model with skew-normal random effects and measurement errors (IG_SNREME): 


$$
\left\{\begin{array}{l}
Y\left(t_{k}\right) \sim I G\left(\beta \Lambda\left(t_{k}\right), \eta \Lambda^{2}\left(t_{k}\right)\right), \beta^{-1} \sim S N\left(\mu, \sigma^{2}, \alpha\right) \\
Z\left(t_{k}\right)=Y\left(t_{k}\right)+\varepsilon\left(t_{k}\right), \varepsilon\left(t_{k}\right) \sim N\left(0, \sigma_{\varepsilon}^{2}\right)
\end{array}\right.
$$

Based on the parameter estimations, we can then obtain the lifetime distribution and reliability assessment for each candidate degradation model. Figure 2 and Table 4 show the lifetime estimation accuracy for the case of perfect measurements. It can be seen that the true model IG_SNRE can be accurately selected with the best fitting performance and the lowest mean squared error (MSE) between the true and estimated curves. The model IG_SNREME can also provide rather good performance. The comparison results for the case of perturbed measurements are shown in Figure 3 and Table 5, which indicate that using the true model IG_SNREME leads to the best estimation performance among all the considered candidate models. Furthermore, for both cases, if we choose the wrong models IG_FE or IG_NRE, then the lifetime and reliability of the product will be either overestimated or underestimated, which will lead to additional life cycle cost because of unexpected failure or premature maintenance actions.

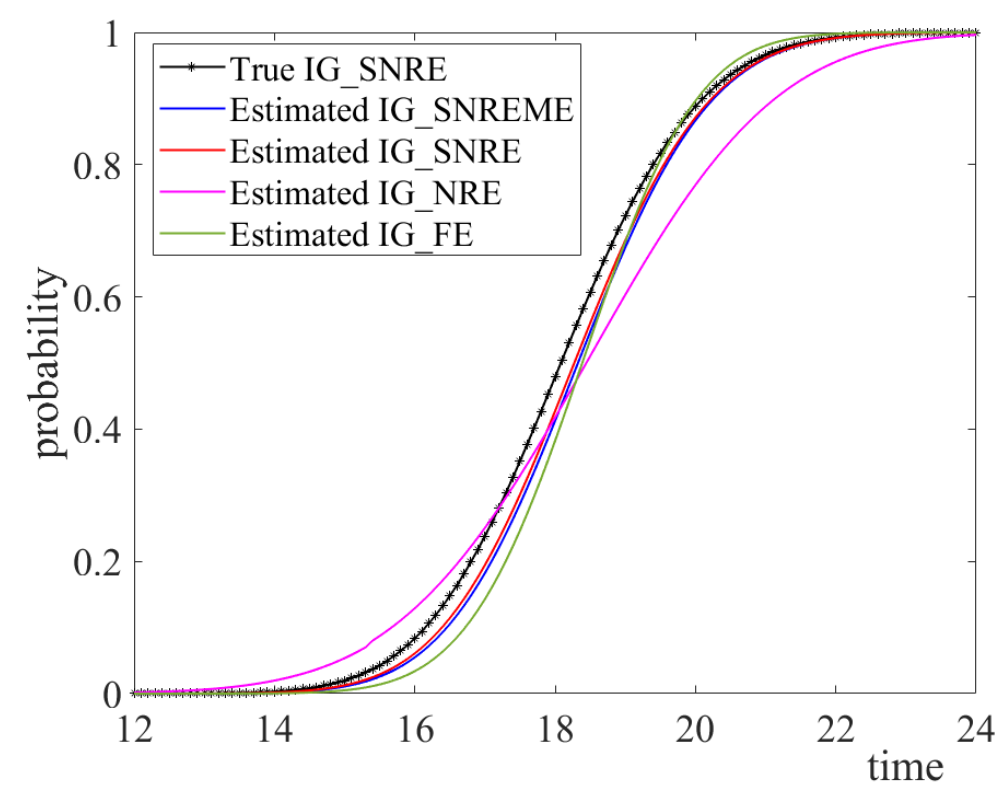

Figure 2 True and estimated lifetime CDFs for simulated degradation data without measurement errors

Table 4 MSEs of candidate models for simulated degradation data without measurement errors

\begin{tabular}{|c|c|}
\hline Candidate Model & MSE \\
\hline IG_FE & $1.91 \mathrm{E}-3$ \\
\hline IG_NRE & $3.62 \mathrm{E}-3$ \\
\hline IG_SNRE & $\mathbf{5 . 6 8 E - 0 4}$ \\
\hline
\end{tabular}




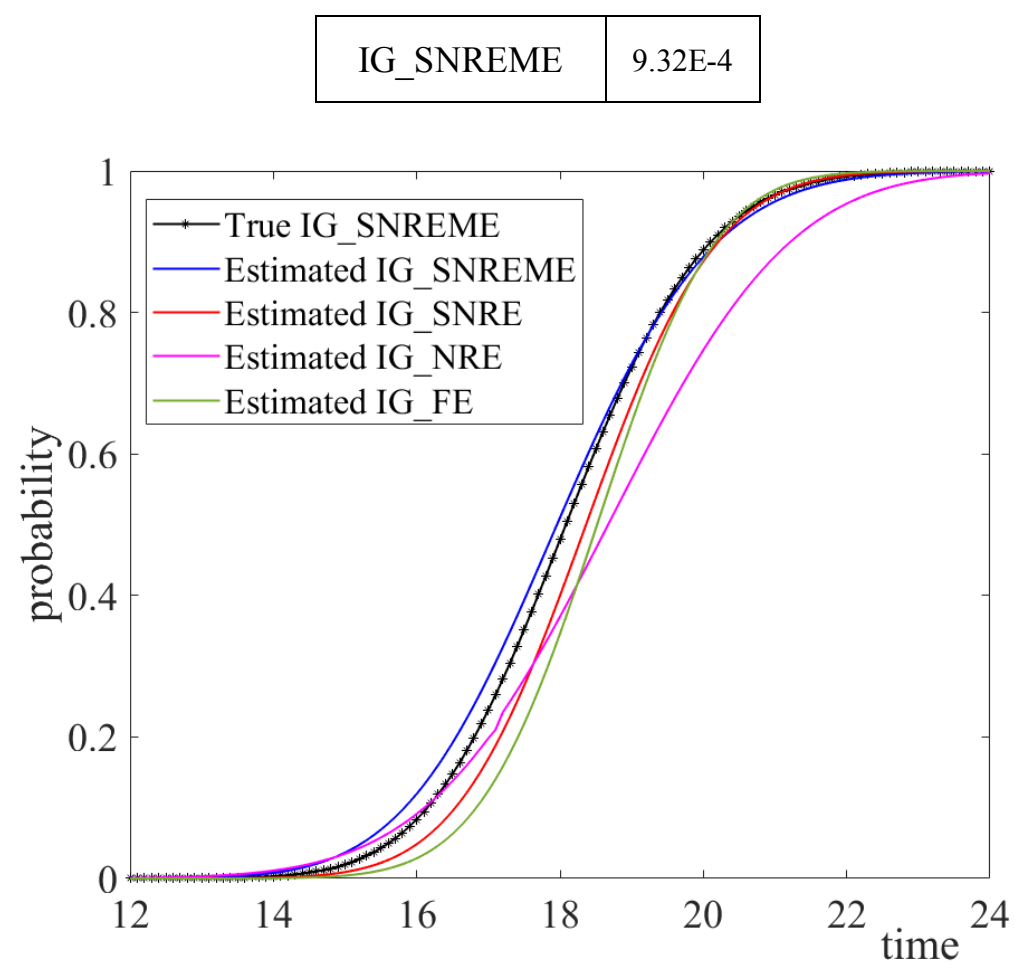

Figure 3 True and estimated lifetime CDFs for simulated degradation data with measurement errors

Table 5 MSEs of candidate models for simulated degradation data with measurement errors

\begin{tabular}{|c|c|}
\hline Candidate Model & MSE \\
\hline IG_FE & $3.36 \mathrm{E}-3$ \\
\hline IG_NRE & $5.63 \mathrm{E}-3$ \\
\hline IG_SNRE & $1.27 \mathrm{E}-03$ \\
\hline IG_SNREME & $\mathbf{4 . 1 3 E - 4}$ \\
\hline
\end{tabular}

\subsection{Application to the GaAs laser degradation data}

Following the analysis in some available works in [1,27,34], here we also assume that the GaAs laser devices experience a linear degradation process, i.e., $\Lambda(t)=t$. Then the MLE method is utilized to estimate the unknown parameters for the 4 considered degradation models, respectively. The estimation results, sample log-likelihoods and the AICs for the 4 candidate models are listed in Table 6 . It can be noticed that compared to the existing models IG_FE and IG_NRE, the proposed models IG_SNRE and IG_SNREME have smaller AICs, which demonstrates that the incorporation of skew-normal random effects can definitely improve the degradation data fitting performance. Furthermore, comparing IG_SNRE and IG_SNREME, we can find that IG_SNREME has a larger sample loglikelihood but also more model parameters, therefore eventually performs worse than IG_SNRE. This illustrates that 
the GaAs laser devices degradation measurements can be considered as perfect (i.e. without any measurement error), and this conclusion is the same as the one in [34] and [48].

Table 6 MLEs, sample log-likelihoods and the AICs of the 4 candidate models for GaAs laser degradation data

\begin{tabular}{|c|c|c|c|c|c|c|c|}
\hline & $\eta$ & $\mu$ & $\sigma$ & $\alpha$ & $\sigma_{\varepsilon}$ & $L$ & AIC \\
\hline IG_FE & $5.43 \mathrm{E}-5$ & 490.65 & - & - & - & 75.03 & -146.06 \\
\hline IG_NRE & $6.09 \mathrm{E}-5$ & 498.59 & 61.3033 & - & - & 75.23 & -144.46 \\
\hline IG_SNRE & $6.09 \mathrm{E}-5$ & 500.26 & 61.41 & -29.70 & - & 77.18 & $\mathbf{- 1 4 6 . 3 6}$ \\
\hline IG_SNREME & $5.99 \mathrm{E}-5$ & 499.25 & 58.27 & -24.52 & $2.778 \mathrm{E}-3$ & 77.33 & -144.66 \\
\hline
\end{tabular}

Moreover, by plugging the degradation parameter estimates into Equations (7), (8) and (13), we have calculated the lifetime CDFs for the candidate models, and have plotted them as lines in Figure 4. Also, the pseudo failure times of the 15 test samples are obtained by regression analysis in [1], and the empirical CDF of the samples is plotted as dots in Figure 4. It can be shown that at the early stage, the lifetime CDF of model IG_NRE is closest to the dots. While in the later stage, it is model IG_SNRE that has closest lifetime CDF. Therefore, to further quantify and compare the performance, we also present the MSE as a distance indicator, and list the MSEs of all candidate models in Table 7. The results indicate that the proposed model IG_SNRE has the smallest MSE, and it provides the most precise estimation for the lifetime distribution.

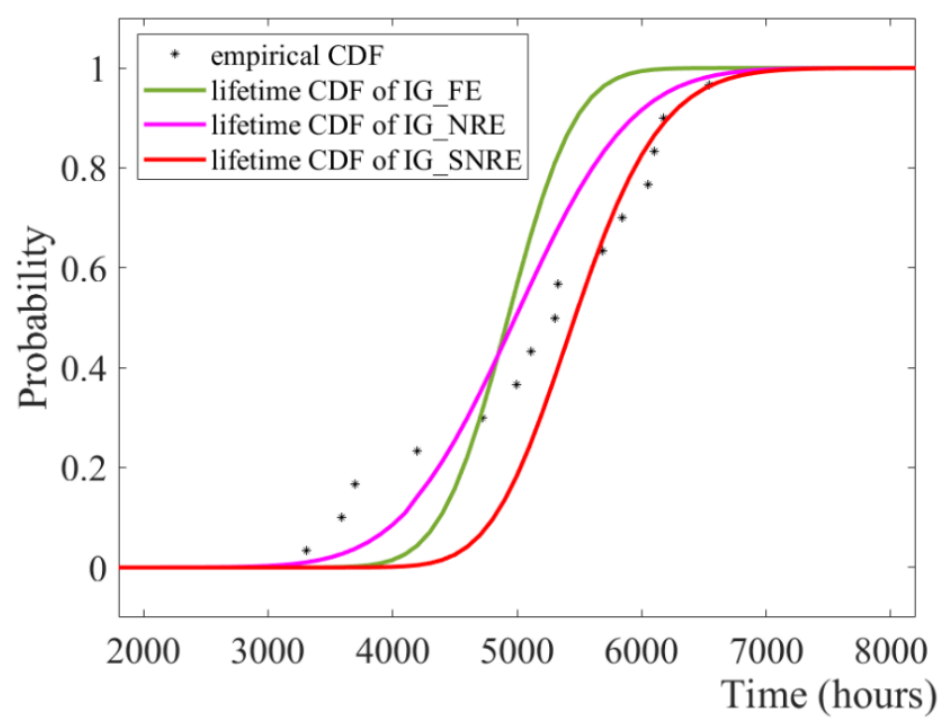

Figure 4 Empirical CDF and estimated lifetime CDFs under candidate models for GaAs laser degradation data 
Table 7 MSEs of candidate models for GaAs laser degradation data

\begin{tabular}{|c|c|}
\hline Candidate Model & MSE \\
\hline IG_FE & $5.21 \mathrm{E}-3$ \\
\hline IG_NRE & $2.22 \mathrm{E}-3$ \\
\hline IG_SNRE & $\mathbf{1 . 6 2 E - 3}$ \\
\hline
\end{tabular}

\subsection{Application to the fatigue crack growth data}

This subsection studies the example of fatigue crack growth data to illustrate the necessity of considering measurement errors. The experiment was carried to explore the degradation law of 2024-T351 aluminum alloy [49], where a constant amplitude fatigue test was conducted to stimulate the fatigue crack growth of 30 specimens. The crack lengths of the samples are measured after 10000 cycles and then every 5000 cycles. The whole degradation data is presented in [49], and the degradation paths are shown in Figure 5.

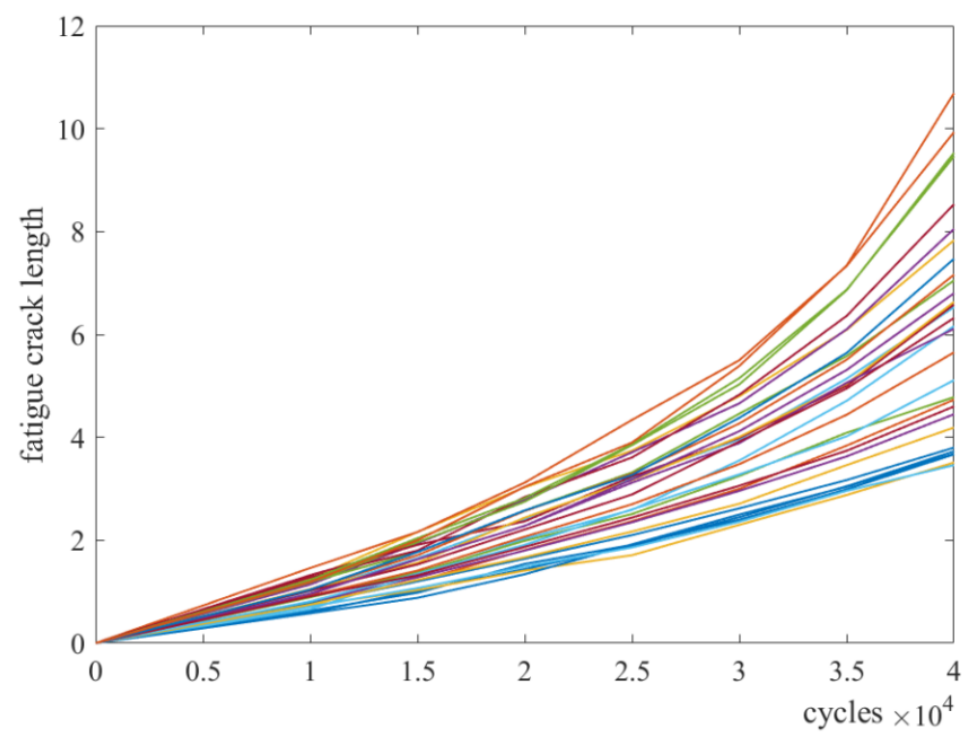

Figure 5 Fatigue crack growth paths of 30 aluminum alloy specimens

An exponential function is found to be the most appropriate to fit the nonlinear degradation path of this crack growth process [27]. Therefore, in this subsection we also choose an exponential drift function, i.e., $\Lambda(t)=e^{\gamma t}-1$. Similar to the GaAs laser example, we firstly fit the degradation path of each specimen with the exponential function, and obtain 30 inverse slopes. Then 5 candidate distribution forms are compared for these inverse slopes, with the loglikelihoods and AICs shown in Table 8. It can be noticed that the skew-normal distribution has the smallest AIC and performs the best to model the random effects of the fatigue data. 
Table 8 Log_likelihoods and AICs for the inverse slopes of fatigue crack growth data

\begin{tabular}{|c|c|c|}
\hline Distribution type for random effects & $\hat{L}$ & AIC \\
\hline Normal & 24.6104 & -45.2208 \\
\hline Gamma & 25.4566 & -46.9132 \\
\hline Lognormal & 24.3753 & -44.7506 \\
\hline Weibull & 25.5983 & -47.1966 \\
\hline Skew-normal & $\mathbf{2 6 . 8 7 7 5}$ & $\mathbf{- 4 7 . 7 5 5}$ \\
\hline
\end{tabular}

We then use the 4 candidate degradation models to estimate degradation parameters for these crack growth data. Table 9 shows the MLEs, sample log-likelihoods and the AICs for the 4 models. It can be seen that model IG_SNREME, i.e., the proposed EIG model with both skew-normal random effects and measurement errors, has the smallest AIC, which demonstrates the necessity of considering measurement errors in the IG process model.

Table 9 MLEs, sample log-likelihoods and the AICs of the 4 candidate models for fatigue crack growth data

\begin{tabular}{|c|c|c|c|c|c|c|c|c|}
\hline & $\eta$ & $\mu$ & $\sigma$ & $\alpha$ & $\gamma$ & $\sigma_{\varepsilon}$ & $L$ & AIC \\
\hline IG_FE & 92.69 & 0.2915 & - & - & 0.2582 & - & -41.4935 & 88.99 \\
\hline IG_NRE & 204.17 & 0.2829 & 0.06558 & - & 0.2428 & - & -37.0137 & 82.03 \\
\hline IG_SNRE & 136.73 & 0.3093 & 0.07698 & 0.8252 & 0.2680 & - & -33.7023 & 77.40 \\
\hline IG_SNREME & 186.55 & 0.2805 & 0.07033 & 0.8481 & 0.2509 & $2.54 \mathrm{E}-4$ & $\mathbf{- 3 2 . 6 3 2 4}$ & $\mathbf{7 7 . 2 6}$ \\
\hline
\end{tabular}

Furthermore, the test specimen is declared to fail when its fatigue crack length exceeds 15 , therefore we can derive the pseudo failure times based on exponential regression results, and then present the empirical CDF of the 30 specimens as dots in Figure 4. Besides, the lifetime CDFs for the candidate models are also plotted in Figure 6. And the MSE of each candidate model with the empirical CDF is provided in Table 10, which indicates that the proposed model IG_SNREME can estimate the lifetime distribution and assess the reliability with the maximum precision, and it is significant and effective to consider measurement errors in the IG process model. 


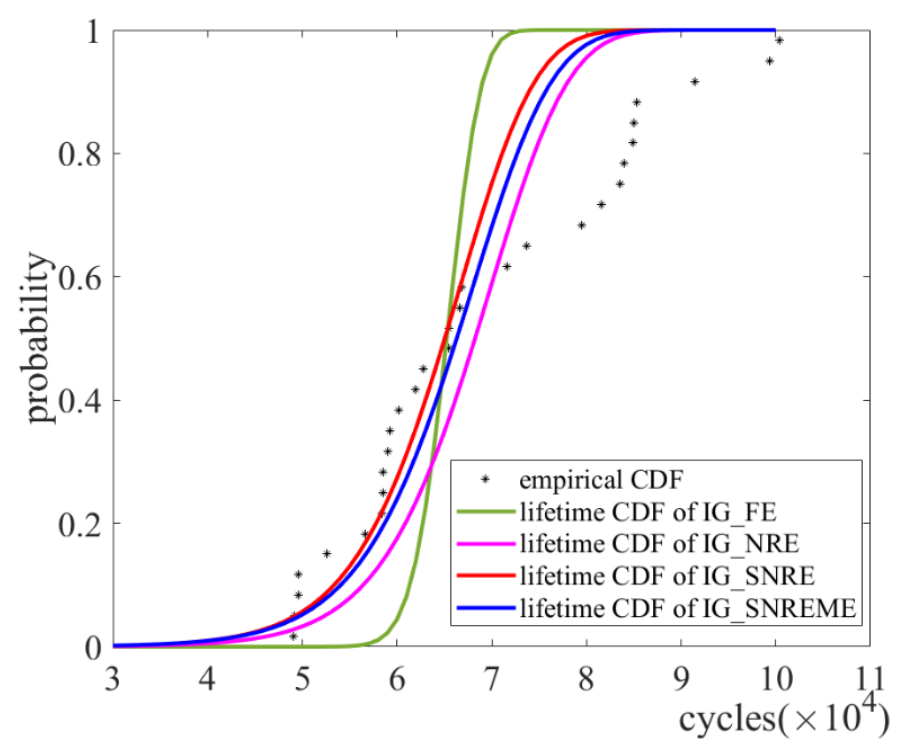

Figure 6 Empirical CDF and estimated lifetime CDFs under candidate models for fatigue crack growth data

Table 10 MSEs of candidate models for fatigue crack growth data

\begin{tabular}{|c|c|}
\hline Candidate Model & MSE \\
\hline IG_FE & $4.53 \mathrm{E}-3$ \\
\hline IG_NRE & $2.14 \mathrm{E}-3$ \\
\hline IG_SNRE & $1.70 \mathrm{E}-3$ \\
\hline IG_SNREME & $\mathbf{1 . 6 6} \mathbf{E - 3}$ \\
\hline
\end{tabular}

\section{Conclusion}

Confronted with degradation processes with asymmetric and non-normal degradation rates, we extend the traditional IG process by incorporating skew-normal random effects, and propose an EIG process model. Reliability assessment and lifetime evaluation of the EIG process model are obtained analytically. Relevant degradation model parameter estimation methods are developed for two scenarios, including the MLEs for perfect measurements via GA, and an extended MC integration method for the MLEs for perturbed measurements. Through simulation studies, we illustrate the effectiveness of the proposed MLEs, and discuss the effect of model mis-specification on lifetime estimation accuracy. Furthermore, by comparing with existing IG process models, the advantage of the proposed EIG process model is demonstrated through two applications of GaAs laser degradation process and fatigue crack growth data. The necessity of considering skew-normal random effects in the IG process model is shown in both examples. Besides, the results show that the laser degradation measurements can be considered as perfect, while the 
measurement error term in the fatigue crack growth model cannot be ignorable. In conclusion, it is significant and effective to consider skew-normal random effects and measurement errors in the IG process model, and we recommend the proposed EIG process models in both degradation data fitting and lifetime evaluation.

For interesting directions of future work, initial degradation values and covariates can be further considered in the EIG process model. Besides, accelerated degradation test planning based on the proposed model can be also studied in the future. Another possible direction can be the optimization of inspection and maintenance strategy for products experiencing the proposed degradation process.

\section{Acknowledgment}

This work was supported by the National Natural Science Foundation of China under Grant 71672006, the Grant JSZL2017601B006, the Fundamental Research Funds for the Central Universities under Grant No. YWF-19-BJ-J160, and the Academic Excellence Foundation of BUAA for PhD Students.

\section{Appendix}

\section{A. Proof of Lemma 1}

Considering that the integral result only depends on $a_{1}, b_{1}, a_{2}, b_{2}$, we can denote it by $I\left(a_{1}, a_{2}, b_{1}, b_{2}\right)$. And due to Dominated Convergence Theorem, we can first compute the second order partial derivative of $I\left(a_{1}, a_{2}, b_{1}, b_{2}\right)$ with respect to $a_{1}, a_{2}$ :

$$
\begin{aligned}
\frac{\partial I\left(a_{1}, a_{2}, b_{1}, b_{2}\right)}{\partial a_{1} \partial a_{2}} & =\int_{-\infty}^{+\infty} \phi\left(a_{1}+b_{1} x\right) \phi\left(a_{2}+b_{2} x\right) \phi(x) d x \\
& =\int_{-\infty}^{+\infty}(2 \pi)^{-\frac{3}{2}} \exp \left\{-\frac{1}{2}\left[\left(a_{1}+b_{1} x\right)^{2}+\left(a_{2}+b_{2} x\right)^{2}+x^{2}\right]\right\} d x \\
& =(2 \pi)^{-\frac{3}{2}} \int_{-\infty}^{+\infty} \exp \left\{-\frac{1}{2}\left[\left(1+b_{1}^{2}+b_{2}^{2}\right) x^{2}+2\left(a_{1} b_{1}+a_{2} b_{2}\right) x+a_{1}^{2}+a_{2}^{2}\right]\right\} d x \\
& =(2 \pi)^{-\frac{3}{2}} \exp \left\{-\frac{1}{2}\left[a_{1}^{2}+a_{2}^{2}-\frac{\left(a_{1} b_{1}+a_{2} b_{2}\right)^{2}}{1+b_{1}^{2}+b_{2}^{2}}\right]\right\} \times \int_{-\infty}^{+\infty} \exp \left\{-\frac{1}{2}\left(1+b_{1}^{2}+b_{2}^{2}\right)\left(x+\frac{a_{1} b_{1}+a_{2} b_{2}}{1+b_{1}^{2}+b_{2}^{2}}\right)^{2}\right\} d x
\end{aligned}
$$

Considering that the integrand function is proportional to the PDF of a normal distributed variable $N\left(-\frac{a_{1} b_{1}+a_{2} b_{2}}{1+b_{1}^{2}+b_{2}^{2}}, \frac{1}{1+b_{1}^{2}+b_{2}^{2}}\right)$, it can be further derived that

$$
\frac{\partial I\left(a_{1}, a_{2}, b_{1}, b_{2}\right)}{\partial a_{1} \partial a_{2}}=\frac{1}{2 \pi\left(1+b_{1}^{2}+b_{2}^{2}\right)} \exp \left\{-\frac{1}{2}\left[a_{1}^{2}+a_{2}^{2}-\frac{\left(a_{1} b_{1}+a_{2} b_{2}\right)^{2}}{1+b_{1}^{2}+b_{2}^{2}}\right]\right\}
$$


Then integrating it over $a_{1}, a_{2}$, we can obtain that

$$
\begin{aligned}
I\left(a_{1}, a_{2}, b_{1}, b_{2}\right) & =\frac{1}{2 \pi\left(1+b_{1}^{2}+b_{2}^{2}\right)} \int_{-\infty}^{a_{1}} \int_{-\infty}^{a_{2}} \exp \left\{-\frac{1}{2}\left[x^{2}+y^{2}-\frac{\left(b_{1} x+b_{2} y\right)^{2}}{1+b_{1}^{2}+b_{2}^{2}}\right]\right\} d x d y \\
& =\frac{1}{2 \pi\left(1+b_{1}^{2}+b_{2}^{2}\right)} \int_{-\infty}^{a_{1}} \int_{-\infty}^{a_{2}} \exp \left\{-\frac{\left(1+b_{2}^{2}\right) x^{2}+\left(1+b_{1}^{2}\right) y^{2}-2 b_{1} b_{2} x y}{2\left(1+b_{1}^{2}+b_{2}^{2}\right)}\right\} d x d y
\end{aligned}
$$

Making the variable substitution $u=\frac{x}{\sqrt{\left(1+b_{1}^{2}\right)}}, v=\frac{y}{\sqrt{\left(1+b_{2}^{2}\right)}}$, then it can be derived that

$$
\begin{aligned}
& I\left(a_{1}, a_{2}, b_{1}, b_{2}\right)=\frac{1}{2 \pi\left(1+b_{1}^{2}+b_{2}^{2}\right)} \int_{-\infty}^{\frac{a_{1}}{\sqrt{\left(1+b_{1}^{2}\right)}}} \int_{-\infty}^{\frac{a_{2}}{\sqrt{\left(1+b_{2}^{2}\right)}}} \exp \left\{-\frac{u^{2}+v^{2}-2 b_{1} b_{2} u v}{2 \frac{1+b_{1}^{2}+b_{2}^{2}}{\left(1+b_{1}^{2}\right)\left(1+b_{2}^{2}\right)}}\right\} \sqrt{\left(1+b_{1}^{2}\right)} d u\left(1+b_{2}^{2}\right) d v
\end{aligned}
$$

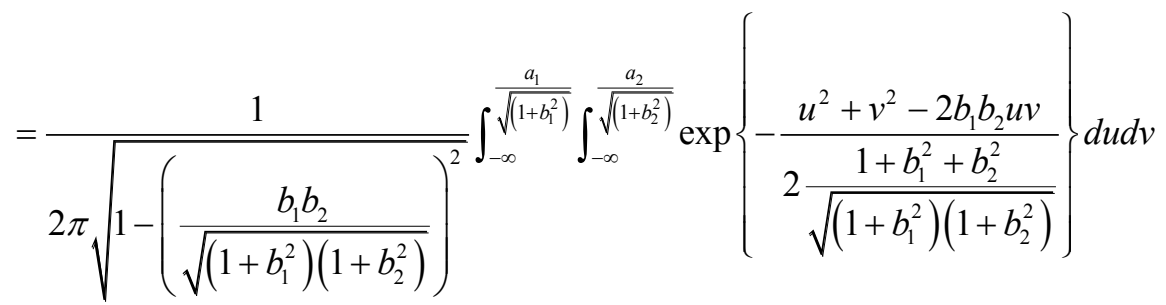

Note that this is just the CDF of the standard bivariate normal distribution, i.e., $\Phi_{2}\left(\frac{a_{1}}{\sqrt{1+b_{1}^{2}}}, \frac{a_{2}}{\sqrt{1+b_{2}^{2}}}, \frac{b_{1} b_{2}}{\sqrt{\left(1+b_{1}^{2}\right)\left(1+b_{2}^{2}\right)}}\right)$, which completes the proof of Lemma 1.

\section{B. Proof of Theorem 2}

Based on some algebraic manipulations, we can obtain:

$$
\begin{aligned}
& E_{Z}\left[\exp \left(A Z+B Z^{2}\right)\right] \\
& =\int_{-\infty}^{+\infty} e^{A z+B z^{2}} \frac{2}{\sqrt{2 \pi} \sigma^{2}} \Phi\left(\alpha \frac{z-\mu}{\sigma}\right) e^{-(z-\mu)^{2} / 2 \sigma^{2}} d z \\
& =\frac{2}{\sqrt{2 \pi} \sigma} \int_{-\infty}^{+\infty} \Phi\left(\alpha \frac{z-\mu}{\sigma}\right) \exp \left\{-\frac{1-2 B \sigma^{2}}{2 \sigma^{2}}\left(z-\frac{A \sigma^{2}+\mu}{1-2 B \sigma^{2}}\right)^{2}+\frac{\left(A \sigma^{2}+\mu\right)^{2}}{1-2 B \sigma^{2}}-\frac{\mu^{2}}{2 \sigma^{2}}\right\} d z \\
& =\frac{2}{\sqrt{2 \pi} \sigma} \exp \left(\frac{2 B \mu^{2}+A^{2} \sigma^{2}+2 A \mu}{2\left(1-2 B \sigma^{2}\right)}\right) \int_{-\infty}^{+\infty} \Phi\left(\alpha \frac{z-\mu}{\sigma}\right) \exp \left\{-\frac{1-2 B \sigma^{2}}{2 \sigma^{2}}\left(z-\frac{A \sigma^{2}+\mu}{1-2 B \sigma^{2}}\right)^{2}\right\} d z
\end{aligned}
$$

Making the variable substitution $z^{\prime}=\sqrt{\frac{1-2 B \sigma^{2}}{\sigma^{2}}}\left(z-\frac{A \sigma^{2}+\mu}{1-2 B \sigma^{2}}\right)$, then it can be derived that 


$$
\begin{aligned}
E_{Z}\left[\exp \left(A Z+B Z^{2}\right)\right] & =\frac{2}{\sqrt{1-2 B \sigma^{2}}} \exp \left(\frac{2 B \mu^{2}+A^{2} \sigma^{2}+2 A \mu}{2\left(1-2 B \sigma^{2}\right)}\right) \int_{-\infty}^{+\infty} \Phi\left(\frac{\alpha}{\sqrt{1-2 B \sigma^{2}}} z^{\prime}+\frac{\alpha \sigma(A+2 B \mu)}{1-2 B \sigma^{2}}\right) \phi\left(z^{\prime}\right) d z^{\prime} \\
& =\frac{2}{\sqrt{1-2 B \sigma^{2}}} \exp \left(\frac{2 B \mu^{2}+A^{2} \sigma^{2}+2 A \mu}{2\left(1-2 B \sigma^{2}\right)}\right) \times \Phi\left(\frac{\alpha \sigma(A+2 B \mu)}{\left(1-2 B \sigma^{2}\right)^{2}+\alpha^{2}\left(1-2 B \sigma^{2}\right)}\right)
\end{aligned}
$$

The last equation is satisfied because of the corollary that

$$
\int_{-\infty}^{+\infty} \Phi\left(a_{1}+b_{1} x\right) \phi(x) d x=\Phi\left(\frac{a_{1}}{\sqrt{1+b_{1}^{2}}}\right), a_{1}, b_{1} \in R
$$

which can be obtained by setting $a_{2}=b_{2}=+\infty$ in Lemma 1 . And this completes the proof of Theorem 2 .

\section{Reference}

[1] Meeker WQ, Escobar L. Statistical methods for reliability data. Wiley 1998.

[2] Zhang Z, Si X, Hu C, Kong X. Degradation modeling-based remaining useful life estimation: A review on approaches for systems with heterogeneity. Proceedings of the Institution of Mechanical Engineers, Part O: Journal of Risk and Reliability 2015; 229: 343-355. doi:10.1177/1748006X15579322.

[3] Ye Z-S, Xie M. Stochastic modelling and analysis of degradation for highly reliable products. Applied Stochastic Models in Business and Industry 2015; 31: 16-32. doi:10.1002/asmb.2063.

[4] Ye Z-S, Chen N, Shen Y. A new class of Wiener process models for degradation analysis. Reliability Engineering \& System Safety 2015; 139: 58-67. doi:10.1016/j.ress.2015.02.005.

[5] Zhai Q, Ye Z. RUL Prediction of Deteriorating Products Using an Adaptive Wiener Process Model. IEEE Transactions on Industrial Informatics 2017; 13: 2911-2921. doi:10.1109/TII.2017.2684821.

[6] Le Son K, Fouladirad M, Barros A. Remaining useful lifetime estimation and noisy gamma deterioration process. Reliability Engineering \& System Safety 2016; 149: 76-87. doi:10.1016/j.ress.2015.12.016.

[7] Paroissin C. Online Estimation Methods for the Gamma Degradation Process. IEEE Transactions on Reliability 2017; 66: 1361-1367. doi:10.1109/TR.2017.2757768.

[8] Wang X, Xu D. An Inverse Gaussian Process Model for Degradation Data. Technometrics 2010; 52: 188197. doi:10.1198/TECH.2009.08197.

[9] Ye Z-S, Chen N. The Inverse Gaussian Process as a Degradation Model. Technometrics 2014; 56: 302-311. doi:10.1080/00401706.2013.830074.

[10] Ye Z-S, Chen L-P, Tang LC, Xie M. Accelerated Degradation Test Planning Using the Inverse Gaussian Process. IEEE Transactions on Reliability 2014; 63: 750-763. doi:10.1109/TR.2014.2315773.

[11] Chen N, Ye Z-S, Xiang Y, Zhang L. Condition-based maintenance using the inverse Gaussian degradation model. European Journal of Operational Research 2015; 243: 190-199. doi:10.1016/j.ejor.2014.11.029.

[12] Pan D, Liu J-B, Cao J. Remaining useful life estimation using an inverse Gaussian degradation model. Neurocomputing 2016; 185: 64-72. doi:10.1016/j.neucom.2015.12.041.

[13] Peng W, Li Y-F, Yang Y-J, Zhu S-P, Huang H-Z. Bivariate Analysis of Incomplete Degradation 
Observations Based on Inverse Gaussian Processes and Copulas. IEEE Transactions on Reliability 2016; 65: 624639. doi:10.1109/TR.2015.2513038.

[14] Rodríguez-Picón LA, Rodríguez-Picón AP, Alvarado-Iniesta A. Degradation modeling of 2 fatigue-crack growth characteristics based on inverse Gaussian processes: A case study. Applied Stochastic Models in Business and Industry 2018. doi:10.1002/asmb.2329.

[15] Hong L, Zhai Q, Wang X, Ye Z. System Reliability Evaluation Under Dynamic Operating Conditions. IEEE Transactions on Reliability 2018. doi:10.1109/TR.2018.2869572.

[16] Shen L, Wang Y, Zhai Q, Tang Y. Degradation Modeling Using Stochastic Processes With Random Initial Degradation. IEEE Transactions on Reliability 2018. doi:10.1109/TR.2018.2885133.

[17] Guo J, Wang C, Cabrera J, Elsayed EA. Improved inverse Gaussian process and bootstrap: Degradation and reliability metrics. Reliability Engineering \& System Safety 2018; 178: 269-277. doi:10.1016/j.ress.2018.06.013.

[18] Liu D, Wang S, Zhang C, Tomovic M. Bayesian model averaging based reliability analysis method for monotonic degradation dataset based on inverse Gaussian process and Gamma process. Reliability Engineering \& System Safety 2018; 180: 25-38. doi:10.1016/j.ress.2018.06.019.

[19] Peng W, Li Y-F, Yang Y-J, Huang H-Z, Zuo MJ. Inverse Gaussian process models for degradation analysis: A Bayesian perspective. Reliability Engineering \& System Safety 2014; 130: 175-189. doi:10.1016/j.ress.2014.06.005.

[20] Peng W, Liu Y, Li Y-F, Zhu S-P, Huang H-Z. A Bayesian optimal design for degradation tests based on the inverse Gaussian process. Journal of Mechanical Science and Technology 2014; 28: 3937-3946. doi:10.1007/s12206014-0904-x.

[21] Peng W, Li Y-F, Yang Y-J, Mi J, Huang H-Z. Bayesian Degradation Analysis With Inverse Gaussian Process Models Under Time-Varying Degradation Rates. IEEE Transactions on Reliability 2017; 66: 84-96. doi:10.1109/TR.2016.2635149.

[22] Li X, Hu Y, Zio E, Kang R. A Bayesian Optimal Design for Accelerated Degradation Testing Based on the Inverse Gaussian Process. IEEE Access 2017; 5: 5690-5701. doi:10.1109/ACCESS.2017.2683533.

[23] He D, Wang Y, Chang G. Objective Bayesian analysis for the accelerated degradation model based on the inverse Gaussian process. Applied Mathematical Modelling 2018; 61: 341-350. doi:10.1016/j.apm.2018.04.025.

[24] Hao S, Yang J, Berenguer C. Nonlinear Step-Stress Accelerated Degradation Modelling considering Three Sources of Variability. Reliability Engineering \& System Safety 2017; 172: 207-215. doi:10.1016/j.ress.2017.12.012.

[25] Wang X. Wiener processes with random effects for degradation data. Journal of Multivariate Analysis 2010; 101: 340-351. doi:10.1016/j.jmva.2008.12.007.

[26] Tsai C-C, Tseng S-T, Balakrishnan N. Optimal Design for Degradation Tests Based on Gamma Processes With Random Effects. IEEE Transactions on Reliability 2012; 61: 604-613. doi:10.1109/TR.2012.2194351.

[27] Peng C-Y. Inverse Gaussian Processes With Random Effects and Explanatory Variables for Degradation Data. Technometrics 2015; 57: 100-111. doi:10.1080/00401706.2013.879077.

[28] Zhai Q, Chen P, Hong L, Shen L. A random-effects Wiener degradation model based on accelerated failure time. Reliability Engineering \& System Safety 2018; 180: 94-103. doi:10.1016/j.ress.2018.07.003.

[29] Pan D, Liu J-B, Yang W. A new result on lifetime estimation based on skew-Wiener degradation model. 
Statistics \& Probability Letters 2018; 138: 157-164. doi:10.1016/j.spl.2018.03.009.

[30] Azzalini A. A Class of Distributions Which Includes the Normal Ones. Scandinavian Journal of Statistics 1985; 12: 171-178.

[31] Azzalini A, Valle AD. The multivariate skew-normal distribution. Biometrika 1996; 83: 715-726. doi:10.1093/biomet/83.4.715.

[32] Azzalini A, Capitanio A. Statistical applications of the multivariate skew normal distribution. Journal of the Royal Statistical Society: Series B (Statistical Methodology) 1999; 61: 579-602. doi:10.1111/1467-9868.00194.

[33] Zhang Z, Si X, Hu C, Lei Y. Degradation data analysis and remaining useful life estimation: A review on Wiener-process-based methods. European Journal of Operational Research 2018; 271: 775-796. doi:10.1016/j.ejor.2018.02.033.

[34] Chien-Yu Peng, Sheng-Tsaing Tseng. Statistical Lifetime Inference With Skew-Wiener Linear Degradation Models. IEEE Transactions on Reliability 2013; 62: 338-350. doi:10.1109/TR.2013.2257055.

[35] Wang X, Balakrishnan N, Guo B. Residual Life Estimation Based on a Generalized Wiener Process with Skew-normal Random Effects. Communications in Statistics - Simulation and Computation 2016; 45: 2158-2181. doi:10.1080/03610918.2014.894057.

[36] Huang Z, Xu Z, Ke X, Wang W, Sun Y. Remaining useful life prediction for an adaptive skew-Wiener process model. Mechanical Systems and Signal Processing 2017; 87: 294-306. doi:10.1016/j.ymssp.2016.10.027.

[37] Pan D, Wei Y, Fang H, Yang W. A reliability estimation approach via Wiener degradation model with measurement errors. Applied Mathematics and Computation 2018; 320: 131-141. doi:10.1016/j.amc.2017.09.020.

[38] Kallen MJ, van Noortwijk JM. Optimal maintenance decisions under imperfect inspection. Reliability Engineering \& System Safety 2005; 90: 177-185. doi:10.1016/j.ress.2004.10.004.

[39] Lu D, Pandey MD, Xie W-C. An efficient method for the estimation of parameters of stochastic gamma process from noisy degradation measurements. Proceedings of the Institution of Mechanical Engineers, Part O: Journal of Risk and Reliability 2013; 227: 425-433. doi:10.1177/1748006X13477008.

[40] Pulcini G. A perturbed gamma process with statistically dependent measurement errors. Reliability Engineering \& System Safety 2016; 152: 296-306. doi:10.1016/j.ress.2016.03.024.

[41] Qin H, Zhang S, Zhou W. Inverse Gaussian process-based corrosion growth modeling and its application in the reliability analysis for energy pipelines. Frontiers of Structural and Civil Engineering 2013; 7: 276-287. doi:10.1007/s11709-013-0207-9.

[42] Zhang S, Zhou W, Qin H. Inverse Gaussian process-based corrosion growth model for energy pipelines considering the sizing error in inspection data. Corrosion Science 2013; 73: 309-320. doi:10.1016/j.corsci.2013.04.020.

[43] Chien-Yu Peng, Sheng-Tsaing Tseng. Mis-Specification Analysis of Linear Degradation Models. IEEE Transactions on Reliability 2009; 58: 444-455. doi:10.1109/TR.2009.2026784.

[44] Owen DB. A Table of Normal Integrals. Communications in Statistics - Simulation and Computation 1980; 9: 389-419. doi:10.1080/03610918008812164.

[45] Li J, Wang Z, Zhang Y, Fu H, Liu C, Krishnaswamy S. Degradation data analysis based on a generalized Wiener process subject to measurement error. Mechanical Systems and Signal Processing 2017; 94: 57-72. 
doi:10.1016/j.ymssp.2017.02.031.

[46] Li J, Wang Z, Zhang Y, Liu C, Fu H. A nonlinear Wiener process degradation model with autoregressive errors. Reliability Engineering \& System Safety 2018; 173: 48-57. doi:10.1016/j.ress.2017.11.003.

[47] Wang Z, Li J, Ma X, Zhang Y, Fu H, Krishnaswamy S. A Generalized Wiener Process Degradation Model with Two Transformed Time Scales. Quality and Reliability Engineering International 2017; 33: 693-708. doi:10.1002/qre.2049.

[48] Tsai C-C, Tseng S-T, Balakrishnan N. Mis-specification analyses of gamma and Wiener degradation processes. Journal of Statistical Planning and Inference 2011; 141: 3725-3735. doi:10.1016/j.jspi.2011.06.008.

[49] Wu WF, Ni CC. A study of stochastic fatigue crack growth modeling through experimental data. Probabilistic Engineering Mechanics 2003; 18: 107-118. doi:10.1016/S0266-8920(02)00053-X. 